\title{
Clusterin: A potential target for improving response to antiestrogens
}

\author{
SARA TOFFANIN ${ }^{1}$, MARIA GRAZIA DAIDONE ${ }^{1}$, PATRIZIA MIODINI ${ }^{1}$, \\ LORIS DE CECCO $^{1,2}$, PAOLO GANDELLINI ${ }^{1}$ and VERA CAPPELLETTI ${ }^{1}$ \\ ${ }^{1}$ Department of Experimental Oncology, Fondazione IRCCS Istituto Nazionale dei Tumori; \\ ${ }^{2}$ Molecular Cancer Genetics, Fondazione Istituto FIRC di Oncologia Molecolare, Milan, Italy
}

Received April 15, 2008; Accepted June 18, 2008

\section{DOI: 10.3892/ijo_00000066}

\begin{abstract}
Antiestrogens represent the first line of therapy in the treatment of estrogen receptor-positive $\left(\mathrm{ER}^{+}\right)$breast cancer patients. Unfortunately, up to $40 \%$ of patients develop resistance associated with progression and frequently die for metastatic breast cancer. The molecular events leading to pharmacological resistance are not completely understood. We attempted to verify in an experimental model the role of cytoplasmic clusterin (CLU), a cytoprotective protein found to be up-regulated in antiestrogen-resistant patients, following neoadjuvant treatment with toremifene. The role of cytoplasmic clusterin in modulating response to two antiestrogens (toremifene and tamoxifen) was studied in two $\mathrm{ER}^{+}$anti-estrogen-sensitive cell lines (MCF-7, 734B) and one $\mathrm{ER}^{+}$antiestrogen-resistant cell line (T47D) using siRNA strategy. Resistant cells were characterised by higher levels of cytoplasmic clusterin than sensitive cells, and antiestrogen treatments up-regulated clusterin levels in both sensitive and resistant cell lines. Treatment with siRNA completely abolished cytoplasmic clusterin expression in all cell lines, but its down-regulation resulted in a significant decrease of cell growth only in the resistant line. We therefore concluded that: i) basal clusterin levels are higher in antiestrogen resistant cells, ii) clusterin is up-regulated following antiestrogen treatment independently of the sensitivity of the cell line, iii) downregulation of cytoplasmic clusterin restores sensitivity to toremifene in the antiestrogen-resistant cell line. Such results support the concept that targeting CLU could represent a promising therapeutic strategy in association with antiestrogen treatment in breast cancer patients.
\end{abstract}

\section{Introduction}

Selective estrogen receptor modulators have been the most frequently used treatment for estrogen receptor-positive

Correspondence to: Dr Vera Cappelletti, Department of Experimental Oncology, Fondazione IRCCS Istituto Nazionale dei Tumori, Via Venezian 1, I-20133 Milano, Italy

E-mail: vera.cappelletti@istitutotumori.mi.it

Key words: antiestrogen, breast cancer, clusterin, siRNA, silencing
$\left(\mathrm{ER}^{+}\right)$breast cancer, and their introduction has significantly contributed to the reduction of mortality observed in recent decades. Nonetheless, up to $40 \%$ of patients under treatment develop resistance associated to relapse or disease progression and frequently die from metastatic breast cancer. In most cases, resistance is not associated to loss of the receptor, which still seems to play a role in regulating tumour growth even in resistant tumours. The mechanisms of antiestrogen resistance are complex and frequently due to post-translational modifications of the estrogen receptor that occur as a consequence of activation of other signalling pathways, mainly supported by growth factors (1). Resistance has been traditionally subdivided into de novo (intrinsic) and acquired resistance. Not only the pre-existing molecular pattern of the tumour is important for response to antiestrogen treatment, but early treatment-induced modulations of cellular pathways are important as well. This concept has been recently demonstrated taking advantage of gene-profiling studies associated to a neo-adjuvant clinical trial (2). By profiling samples obtained from a study with primary treatment with toremifene (3), we observed that genes associated with response to treatment and genes whose expression was modulated by the treatment are different and that treatment response affects gene modulation. A higher number of genes was modulated by treatment in non-responders compared to responders including genes likely to represent molecular hurdles for complete response to treatment and which may be targeted for improving response. Among such genes, we focused on clusterin, also known as apolipoprotein $\mathrm{J}$, testosterone-repressed prostate message-2 or sulphated glycoprotein-2. CLU is involved in many physiological processes, and its overexpression is associated with cell survival and apoptosis (4) whose balance markedly influences tumour progression and treatment resistance. The functional ambiguity of this protein is explained by the existence of two splicing variants coding for one prevalently nuclear form (lacking exon II) and one cytoplasmic secreted form respectively associated with apoptosis and survival. In response to cell stress, clusterin is rapidly up-regulated, and the increased nuclear $55 \mathrm{kDa}$ form suggests an apoptotic response, although the precise relationship with the cell death mechanism is not understood. However, other studies have clearly demonstrated that overexpression of clusterin is a cytoprotective, pro-survival response of cells to stress. The 
latter mechanism is also supported by recent findings showing that targeting cytoplasmatic clusterin may interfere with drugresistance processes mediated by cell survival proteins (5).

This approach appears to be particularly helpful in those tissues which overexpress clusterin such as prostate (6), breast (7), and lung (8). In this sense, pioneering studies in prostate cancer showed that the use of antisense nucleotides against clusterin was able to restore androgen - and chemosensitivity. Similarly, in bladder and lung cancer cells, antisense suppression of CLU resulted into a potentiation of antiapoptotic effects by cisplatin and paclitaxel (9) and an effective modulation of clusterin could also be achieved with small interfering RNA molecules (10).

In the present study, we investigated the expression of clusterin at a protein level in response to antiestrogen treatment in three $\mathrm{ER}^{+}$breast cancer cell lines (MCF-7, 734B and T47D) characterised by a different response profile to antiestrogens and the effect of its down-regulation by siRNA strategy on response to the antiestrogens toremifene (TOR) and tamoxifen (TAM).

The finding that down-regulation of clusterin was able to increase sensitivity to antiestrogens especially in the resistant cell line (T47D) might indicate clusterin as a candidate target to improve the response to antiestrogens and delay the appearance of acquired hormone-resistance.

\section{Materials and methods}

Cell line cultures. The human breast cancer cell lines MCF-7, 734B and T47D were cultured in Dulbecco's minimum Eagle's medium/F12, DMEM/F12 (Sigma-Aldrich, St. Louis, MO, USA) without red phenol supplemented with $5 \%$ fetal bovine serum (Cambrex Bioscience, Milan, Italy) at $37^{\circ} \mathrm{C}$ under $5 \% \mathrm{CO}_{2}$. Cells were split at least once before starting the experiments.

In order to analyse the expression level of clusterin following antiestrogen treatments, the three cell lines were seeded in $25-\mathrm{cm}^{2}$ flask for $24 \mathrm{~h}$ and allowed to attach. The day after, the medium was changed with DMEM/F12 with $5 \%$ stripped FBS supplemented with $4 \mathrm{OH}$-toremifene $(4 \mathrm{OH}-$ TOR) and 4OH-tamoxifen (4OH-TAM) $10^{-7} \mathrm{M}$ (SigmaAldrich). Cells were maintained in this medium for 3 days until harvested.

siRNA transfection. For transfection assays MCF-7, 734B, and T47D cells were seeded in DMEM/F12 5\% FBS in $25-\mathrm{cm}^{2}$ flasks and allowed to attach. The next day, the culture medium was aspirated and the cell monolayers were washed with $2 \mathrm{ml}$ of Opti-MEM (Gibco, Carlsbad, CA, USA) to remove all the traces of serum and were preincubated with Lipofectamine 2000 reagent (Invitrogen-Life Technologies Inc., Carlsbad, CA, USA) in serum-free medium Opti-MEM for $20 \mathrm{~min}$ before adding siRNA oligonucleotides $(25 \mathrm{nM})$, separately diluted in the same medium $(3 \mu \mathrm{g} / \mathrm{ml}$ Lipofectamine final concentration).

The custom-synthesised siRNAs (Dharmacon, Research, Inc., Lafayette, CO, USA), were: CLU-V, for the exclusive down-regulation of the clusterin cytoplasmatic form, CLU-III, for the down-regulation of both cytoplasmic and nuclear forms and a scrambled of the BIRC6 gene as control siRNA.
The sequences of the siRNAs were: CLU-III-siRNA, 5'-CUA AUU CAA UAA AAC UGU C $\mathrm{C}_{\mathrm{d}} \mathrm{T}_{\mathrm{d}} \mathrm{T}-3$ ' and 3'dTdTGAU UAA GUU AUU UUG ACA G-5'; CLU-V-siRNA, 5'-AUG AUG AAG ACU CUG CUG C-3' and 3'-UAC UAC UUC UGA GAC GAC G-5'; control siRNA (scrambled of BIRC6 gene), 5'-GCA GUA CAU GGU AUG AUU A $\mathrm{A}_{\mathrm{d}} \mathrm{T}_{\mathrm{d}} \mathrm{T}-3^{\prime}$ and $3{ }^{\prime}{ }_{d} T_{d}$ TCGU CAU GUA CCA UAC UAA U-5'. BLAST analysis showed that CLU-III and CLU-V siRNAs were complementary to sequences of exons 9 and 2, respectively, whereas no homology was found between control siRNA and other known human genes. Since exon 2 is maintained only in the transcript that codifies for cytoplasmic clusterin, CLU-VsiRNA is able to down-regulate only this particular form.

Twenty-four hours after starting the incubation, the medium containing the RNA duplex and Lipofectamine was replaced with DMEM/F12 with 5\% stripped fetal bovine serum (FBS) and cells were treated with antiestrogens. 4OH-TOR and 4OH-TAM were prepared in $2 \times 10^{-3} \mathrm{M}$ stock solutions in ethanol. All the experiments were repeated three times.

Proliferation assay. For proliferation assay, cells were trypsinised, washed once with Dulbecco's phosphate-buffered saline (DPBS) $1 \mathrm{X}$ and harvested. Cells growth was determined by direct counting in a Burker chamber. The viable fraction was determined by trypan-blue exclusion (Sigma-Aldrich). Experiments were carried out in triplicate.

Western blotting. Cytosolic extracts were prepared using a commercially available kit (Active Motif Inc., Carlsbad, CA, USA) according to the manufacturer's instructions. For Western blot analysis, $30 \mu \mathrm{g}$ of cytosolic proteins was separated by SDS-PAGE and transferred onto nitrocellulose membranes. Membranes were blocked for $1 \mathrm{~h}$ in DPBS 1X, 0.1\% Tween-20 with $5 \% \mathrm{w} / \mathrm{v}$ non-fat dry milk and incubated with primary antibody against clusterin (1:500, sc-6419 goat; Santa Cruz Biotechnology, Santa Cruz, CA, USA) and against B-actin (1:1000, 20-33 rabbit; Sigma-Aldrich). Membranes were washed and incubated for $1 \mathrm{~h}$ at room temperature with peroxidase-conjugated anti-goat (Santa Cruz Biotechnology) and anti-rabbit (Amersham Biosciences Europe, Freiburg, Germany) secondary antibodies (1:2000) for the detection of clusterin and $\beta$-actin, respectively. All the antibodies were diluted in DPBS $1 \mathrm{X}, 0.1 \%$ Tween-20 with $5 \%$ w/v non-fat dry milk.

Signals were detected by chemiluminescence reaction (ECL, Amersham Biosciences Europe) according to the manufacturer's instructions. The relative amounts of clusterin protein were quantified by densitometric analysis and normalised with respect to the $\beta$-actin signal.

Statistical analysis. The two-tailed t-test was used to compare the statistical significance of the differences of cell number between all treated samples and controls. Only the significant P-values $(\leq 0.05)$ are indicated. The values are the mean of three independent replicates.

\section{Results}

Biologic model. The three breast cancer cell lines chosen for the study were characterised by different sensitivity profiles 
A

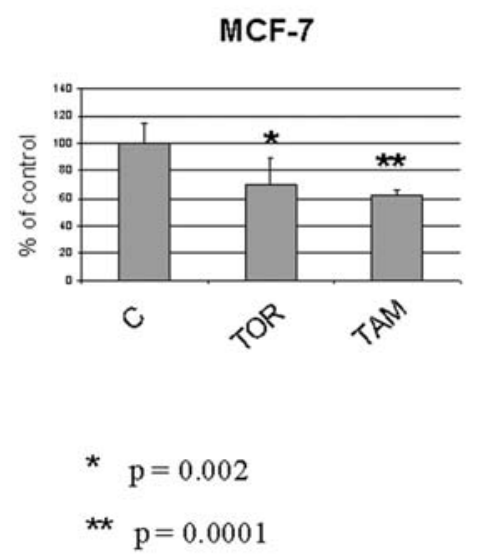

734B

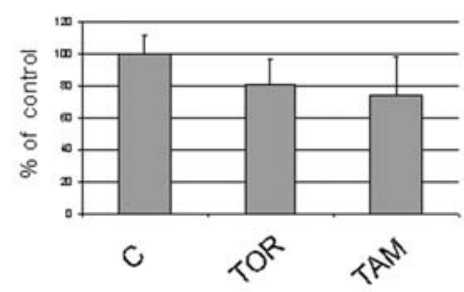

$0 \quad 0^{2}$
T47D

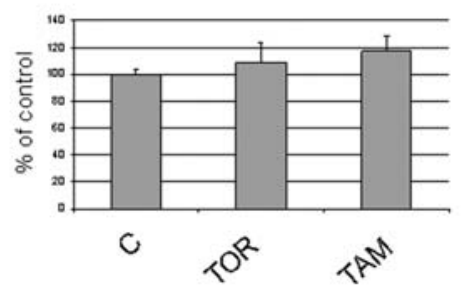

B

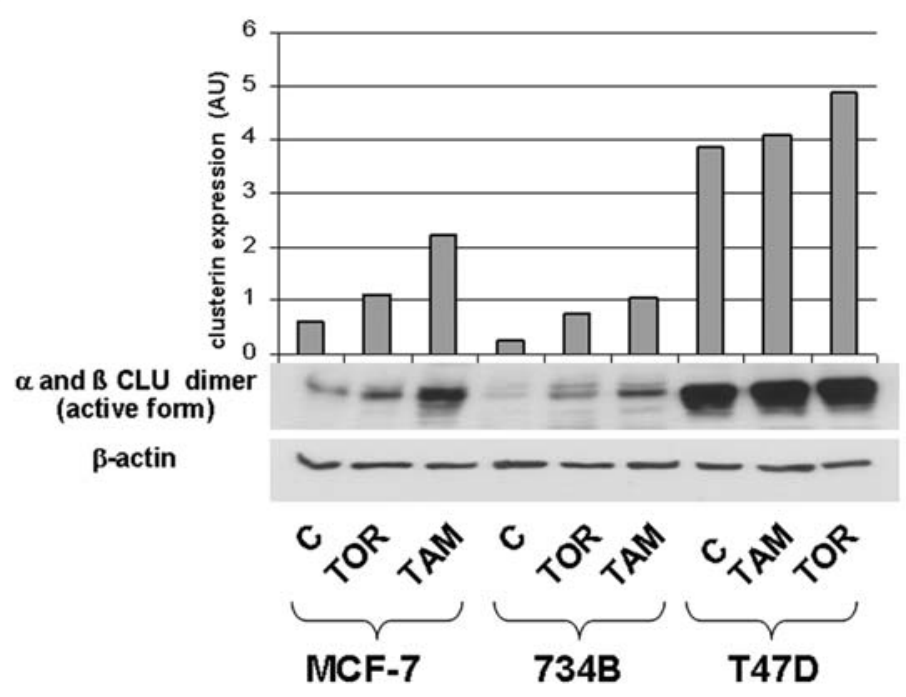

Figure 1. A, Growth assay of three human breast cancer cell lines (MCF-7, 734B and T47D) untreated and treated with $10^{-7} \mathrm{M} 4 \mathrm{OH}-$ toremifene (4OH-TOR) and $4 \mathrm{OH}$-tamoxifen (4OH-TAM) for 3 days. Results are the mean of three independent experiments. Statistical significance: ${ }^{*} \mathrm{P}=0.002$; ${ }^{* *} \mathrm{P}=0.0001$ with respect to the control. B, Western blot analysis of clusterin expression (active form) of MCF-7, 734B and T47D breast cancer cell lines untreated and treated with $10^{-7} \mathrm{M} 4 \mathrm{OH}-\mathrm{TOR}$ and $4 \mathrm{OH}-\mathrm{TAM}$ for 3 days. The relative amount of clusterin was quantified and normalised to the corresponding levels of $\beta$-actin.

towards the two antiestrogens, toremifene and tamoxifen. Indeed, after treatment with $10^{-7} \mathrm{M} 4 \mathrm{OH}-\mathrm{TOR}$ for 3 days in medium supplemented with 5\% FBS, MCF-7 cells diminished to $70 \pm 19 \%$ of the control $(\mathrm{P}=0.002)$ and were defined as sensitive, 734B cells were not significantly inhibited to $81 \pm 15 \%$ and were considered as non-exquisitely sensitive, but still non-resistant, while T47D cells were defined as resistant with a slight up-regulation of growth in the presence of toremifene $(109 \pm 15 \%$ of the control). Similarly, after a treatment with $10^{-7} \mathrm{M} 4 \mathrm{OH}-\mathrm{TAM}$ for 3 days in the same conditions, the growth of MCF-7 and 734B cells was inhibited respectively to $62 \pm 4 \%(\mathrm{P}=0.0001)$ and $74 \pm 24 \%(\mathrm{P}=\mathrm{NS})$ of the control while T47D showed an increase of proliferation $(117 \pm 11 \%$ of the control). The data are shown in Fig. 1A and Table I.

Western blot analysis showed that the two non-resistant cell lines were characterised by lower clusterin protein levels compared to the resistant T47D cell line (Fig. 1B). The basal expression of clusterin was 6 and 15 times greater in T47D
Table I. Cell growth after 3 days of treatment: values represent the percentages of the untreated control.

\begin{tabular}{lccc}
\hline Treatment & MCF-7 & $734 \mathrm{~B}$ & T47D \\
\hline Control & $100 \pm 14$ & $100 \pm 11$ & $100 \pm 4$ \\
Toremifene $10^{-7} \mathrm{M}$ & $70 \pm 19^{\mathrm{a}}$ & $81 \pm 15$ & $109 \pm 15$ \\
Tamoxifen $10^{-7} \mathrm{M}$ & $62 \pm 4^{\mathrm{b}}$ & $74 \pm 24$ & $117 \pm 11$ \\
\hline
\end{tabular}

${ }^{\mathrm{a}} \mathrm{P}=0.02 .{ }^{\mathrm{b}} \mathrm{P}=0.0001$.

than in MCF-7 and 734B, respectively. Furthermore, in all three cell lines clusterin expression increased following antiestrogen treatments, in particular following tamoxifen (4 times higher after tamoxifen treatment in the sensitive cell line MCF-7). 

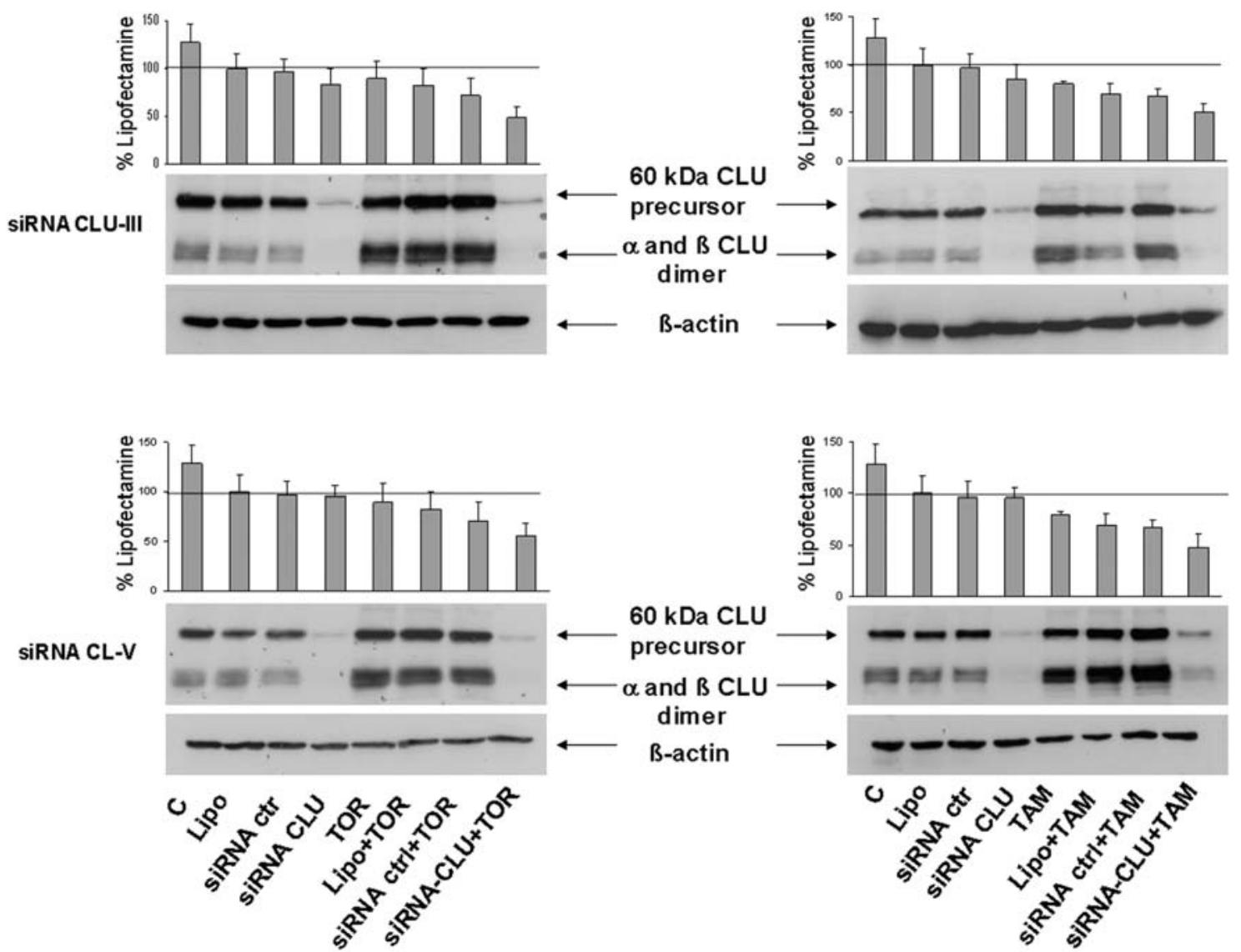

Figure 2. Growth assay of the MCF-7 human breast cancer cell line untransfected or transfected with $25 \mathrm{nM}$ siRNA $\mathrm{CTRL}_{\text {, }}$, siRNA CLU-III (upper part) or siRNA CL-V (lower part) and untreated or treated with $10^{-7} \mathrm{M} 4 \mathrm{OH}-\mathrm{TOR}$ (left part) and 4OH-TAM (right part) for 3 days. Values are the mean of three replicates. A representative Western blotting image is shown for each assay.

Silencing of clusterin. Silencing of clusterin was attempted by RNA interference approach. Specific siRNA designed against distinct exons of the genes were used. Transfection efficiencies were measured for each cell line monitoring green fluorescence protein plasmid internalisation by flow cytometry.

To assess the possible effect of clusterin basal levels and treatment-induced up-regulation of clusterin on sensitivity to antiestrogens, we treated the cells with two different siRNAs targeting distinct exons of the gene: siRNA CLU-III (targeting the secreted anti-apoptotic and the nucler pro-apoptotic form) and siRNA CLU-V (targeting only the secreted form).

The result of the silencing approach was monitored by Western blotting. The $60 \mathrm{kDa}$ clusterin precursor appeared together on the blots, with a protein smear around $40 \mathrm{kDa}$ comprising the two alpha and beta dimers and their relative glycosylated forms.

As seen in Figs. 2 (MCF-7 cells), 3 (734B) and 4 (T47D), siRNA CLU-III and siRNA CLU-V were both able to completely suppress cytoplasmic clusterin protein expression, which showed no recovery at all even after 3 days. However, a slightly less effective silencing was observed in the T47D cell line, which expressed higher CLU protein levels especially after treatment with antiestrogens, which in turn further increased clusterin levels (Fig. 4).

The specificity of the effects exerted by our siRNAs is evident by the absence of any effects on clusterin protein expression when cells were treated with a control siRNA $\left(\right.$ siRNA $\left.A_{\text {CTRL }}\right)$ and by the absence of any effect on $\beta$-actin expression.

Cell growth after clusterin silencing in the non-resistant cell lines. To determine whether reduction of clusterin protein levels affected cell growth in the cell lines alone or in the presence of $10^{-7} \mathrm{M} 4 \mathrm{OH}-\mathrm{TOR}$ (left panel of Figs. 2, 3 and 4) or $10^{-7} \mathrm{M} 4 \mathrm{OH}-\mathrm{TAM}$ (right panel of Figs. 2, 3 and 4), cells were pre-treated with siRNA CLU-III (upper panel Figs. 2, 3 and 4) or siRNA CLU-V (bottom panel Figs. 2, 3 and 4) and grown in the presence of the antiestrogen for 3 days.

Cell growth was assessed by direct cell counting, and to ensure that any effect on growth was in fact correlated to a specific down-regulation of clusterin, the same cells were extracted and used for verification of specific protein down-regulation. Table II reports the data regarding the proliferation assays for the three lines.

Cell growth results were expressed as percentages of the control (i.e., cells treated with the transfecting agent lipofectamine alone, but the untreated control is also reported) using the mean values of at least three separate experiments.

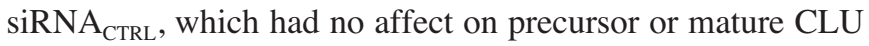
$\alpha$ and $\beta$ isoforms, did not affect the growth of MCF-7 cells in the absence or in the presence of toremifene or tamoxifen. As shown in Table II, neither siRNA CLU-III or CLU-V significantly inhibited growth of $\mathrm{MCF}-7$ cells in the presence 


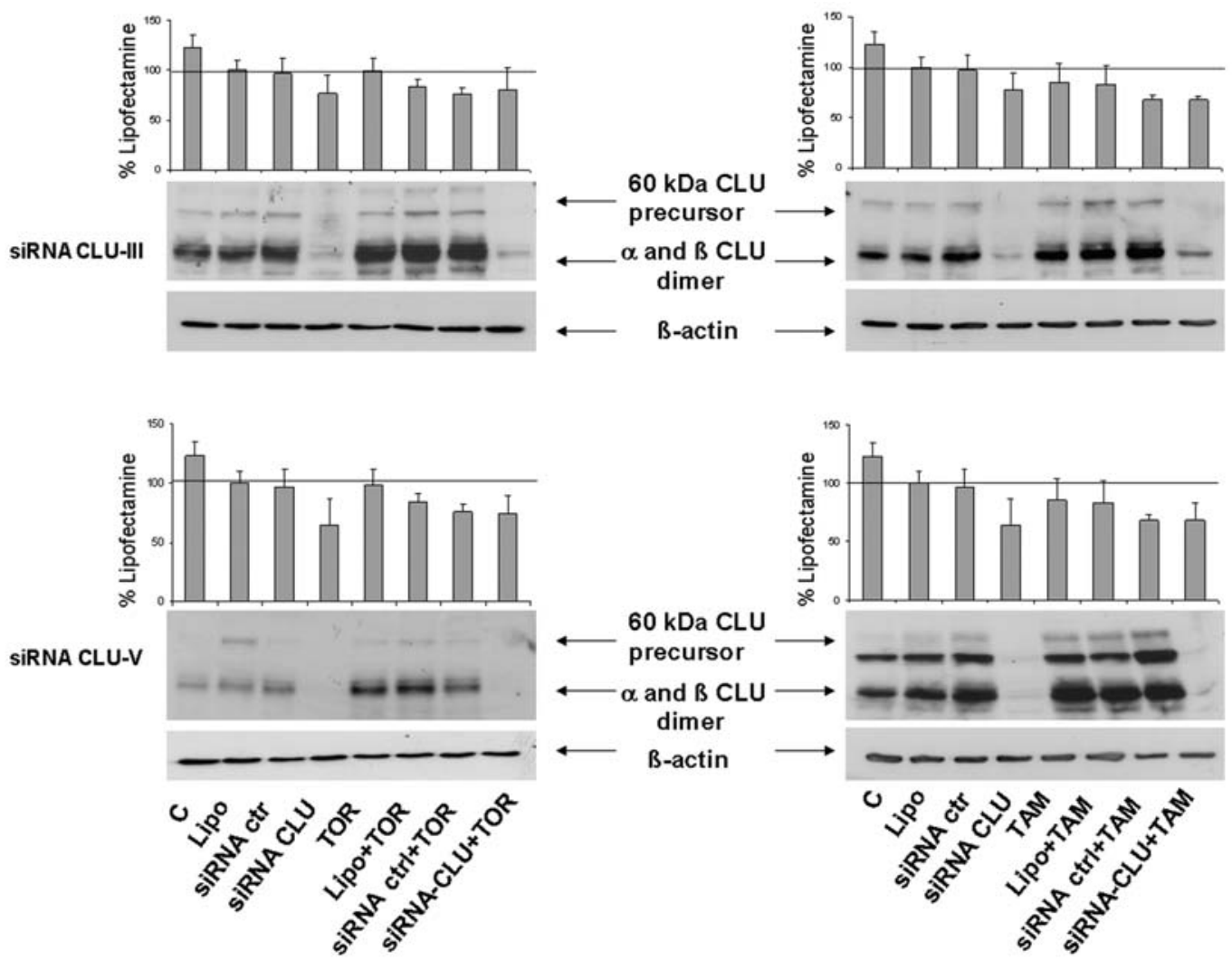

Figure 3. Growth assay of the 734B human breast cancer cell line untransfected or transfected with $25 \mathrm{nM}$ siRNA $\mathrm{CTRL}_{\mathrm{C} L}$, siRNA CLU-III (upper part) or siRNA CL-V (lower part) and untreated or treated with $10^{-7} \mathrm{M} 4 \mathrm{OH}-\mathrm{TOR}$ (left part) and 4OH-TAM (right part) for 3 days. Values are mean of three replicates. A representative Western blotting image is shown for each assay.
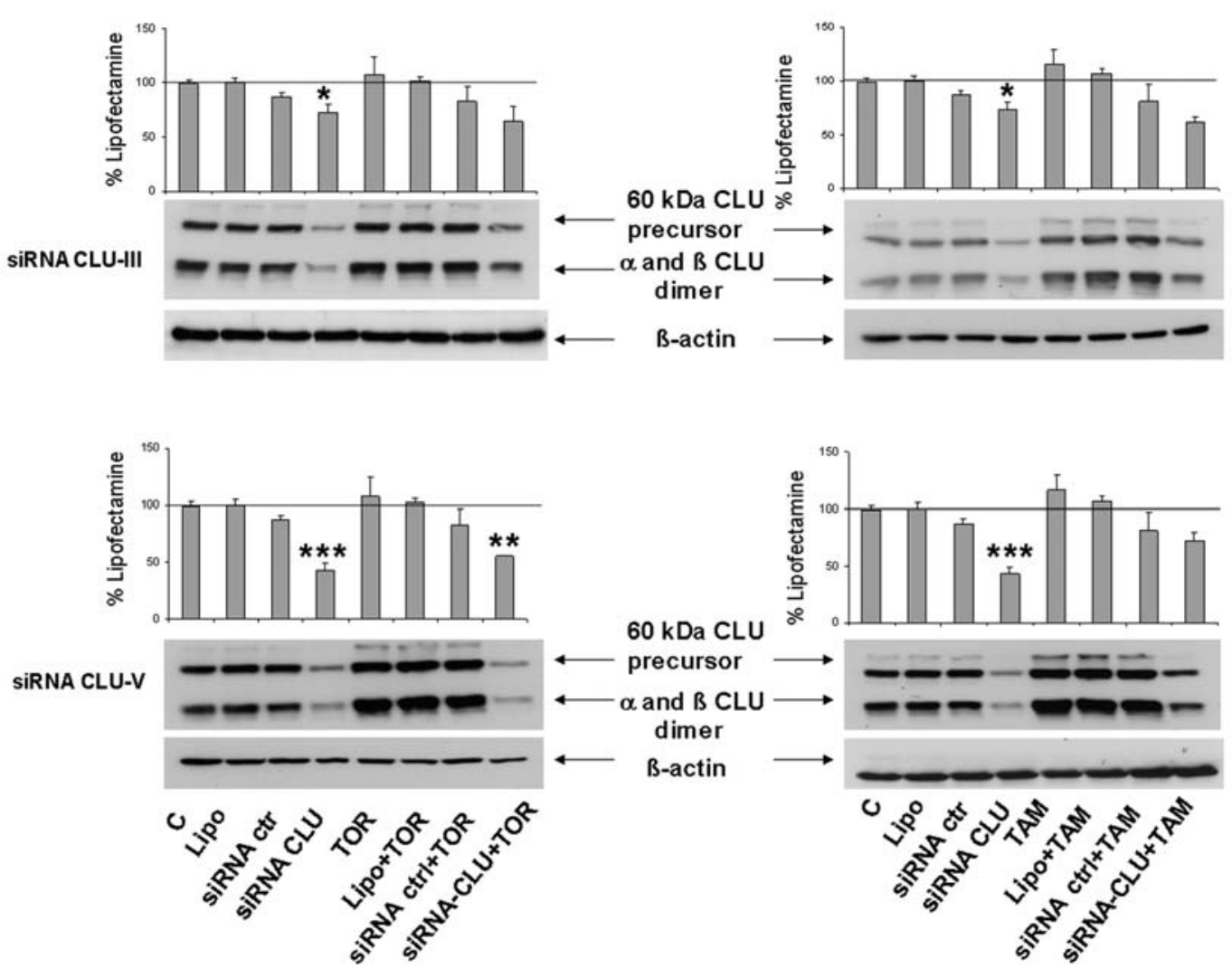

Figure 4. Growth assay of the T47D human breast cancer cell line untransfected or transfected with $25 \mathrm{nM}$ siRNA $\mathrm{CTRL}_{\text {, }}$, siRNA CLU-III (upper part) or siRNA CL-V (lower part) and untreated or treated with $10^{-7} \mathrm{M} 4 \mathrm{OH}-\mathrm{TOR}$ (left part) and 4OH-TAM (right part) for 3 days. Values are mean of three replicates. A representative Western blotting image is shown for each assay. Statistical significance: ${ }^{*} \mathrm{P}=0.046$ and ${ }^{* * * *} \mathrm{P}=0.0001$ with respect to samples treated with siRNA $\mathrm{CTRL}$; ${ }^{* *} \mathrm{P}=0.032$ with respect to samples treated with siRNA $\mathrm{CTRL}_{\mathrm{C}}$ and toremifene. 
Table II. Cell growth after treatment with siRNAs and antiestrogens: values represent the percentages of Lipofectaminetreated cells.

\begin{tabular}{lccl}
\hline Treatment & MCF-7 & $734 \mathrm{~B}$ & T47D \\
\hline Control & $100 \pm 17$ & $100 \pm 10$ & $100 \pm 5$ \\
siRNA ctr & $97 \pm 14$ & $97 \pm 15$ & $87 \pm 4$ \\
siRNA CL-III & $84 \pm 16$ & $77 \pm 18$ & $73 \pm 7^{\mathrm{a}}$ \\
siRNA CL-V & $96 \pm 10$ & $64 \pm 23$ & $43 \pm 6^{\mathrm{b}}$ \\
Toremifene 10-7 M & $82 \pm 17$ & $84 \pm 7$ & $102 \pm 4$ \\
siRNA ctr + TOR & $71 \pm 19$ & $76 \pm 6$ & $83 \pm 14$ \\
siRNA CL-III + TOR & $48 \pm 12$ & $80 \pm 22$ & $64 \pm 14$ \\
siRNA CL-V + TOR & $57 \pm 12$ & $74 \pm 16$ & $56 \pm 0.6^{\mathrm{c}}$ \\
Tamoxifen 10-7 M & $69 \pm 12$ & $85 \pm 19$ & $107 \pm 4$ \\
siRNA ctr + TAM & $67 \pm 7$ & $83 \pm 19$ & $81 \pm 16$ \\
siRNA CL-III + TAM & $50 \pm 9$ & $68 \pm 5$ & $62 \pm 5$ \\
siRNA CL-V + TAM & $48 \pm 12$ & $68 \pm 14$ & $72 \pm 7$ \\
\hline
\end{tabular}

${ }^{\mathrm{a}} \mathrm{P}=0.046$ with respect to $\mathrm{siRNA} \mathrm{CTRL}_{\mathrm{CT}} \cdot{ }^{\mathrm{b}} \mathrm{P}=0.0001$ with respect to siRNA $_{\text {CTRL }} \cdot{ }^{c} \mathrm{P}=0.032$ with respect to siRNA $\mathrm{CTRL}$ in combination with TOR.

or in the absence of treatment, despite complete downregulation of the protein.

Fig. 3 reports the results for the 734B cells. Once again, Western blotting results confirmed the absence of any effects on clusterin protein levels after treatment of the cells with siRNA $_{\text {CTRL, }}$, whereas a complete down-regulation of clusterin was obtained with both tested target siRNAs. Similarly to that observed with MCF-7 cells, both antiestrogens triggered an up-regulation of clusterin which was, however, completely abolished by the silencing procedure. No significant effects on cell growth was observed upon treatment with siRNA $\mathrm{CTRL}_{\mathrm{C}}$, but even the two target siRNAs did not significantly affect cell growth in the absence or in the presence of antiestrogens. As a consequence, the down-regulation of clusterin in MCF-7 and 734B, which express low levels of the protein, did not influence cell growth and did not increase sensitivity to antiestrogens.

Cell growth after clusterin silencing in the resistant cell line. Fig. 4 shows the same type of experiments in the case of the T47D antiestrogen resistant cell line. Basal expression levels of clusterin were very high and further increased after treatment with toremifene and tamoxifen. Similarly to that observed in the two hormone-sensitive cell lines, no interference of control siRNA was observed on protein expression or on growth. However, down-regulation of clusterin affected cell growth. In the presence of siRNA CLU-III, cell growth dropped to $73 \%$ with respect to siRNA $\mathrm{CTRL}_{\mathrm{L}}(\mathrm{P}=0.046)$, whereas in the presence of siRNA CLU-V it dropped to $43 \%$ of the control $(\mathrm{P}=0.0001)$.

Such growth down-regulation exerted by siRNAs was also maintained in the presence of toremifene despite the fact that antiestrogens up-regulate clusterin. When the cells were treated with antiestrogens, neither toremifene nor tamoxifen inhibited cell growth but rather caused a small stimulation. However, when cells were subjected to cytoplasmic clusterin silencing (through treatment with siRNA CLU-V), toremifene was able to cause cell growth inhibition down to $56 \%$ of control, represented by cells treated with toremifene and $\operatorname{siRNA}_{\text {CTRL }}(\mathrm{P}=0.032)$.

\section{Discussion}

Antiestrogens represent the first-line therapy in $\mathrm{ER}^{+}$breast cancer, but unfortunately about $40 \%$ of patients do not respond or develop resistance, determining the failure of the therapy. Although different mechanisms have been proposed to explain the phenomena of resistance, its relatively frequent occurrence represents a challenge for clinicians and still is not completely understood.

Different survival proteins, which are up-regulated after cytostatic and cytotoxic treatments, have been considered as potential targets to overcome the problem of resistance in breast cancer, among them members of the BCL-2 protein family, survivin, heat-shock proteins, Akt and clusterin. One major bottleneck in understanding the mechanism of tamoxifen resistance is the lack of clinical study material, since tumour tissues treated with antiestrogens associated with response information are difficult to obtain, especially for gene expression profiling.

In the present study, we chose to focus on the role of clusterin in determining responsivity to antiestrogens based on results we had previously obtained in a clinical setting (2). By profiling breast tumour samples obtained from $\mathrm{ER}^{+}$breast cancer patients before and after treatment with neoadjuvant toremifene, we observed that the treatment-modulated gene expression varied as a function of clinical response. In particular, many more genes were modulated in patients with a poor response to the treatment than in those with an objective clinical response. We speculated that among genes exclusively modulated in non-responding patients, some may reveal pathways involved in activation of compensative survival/cytoprotective pathways or in resistance mechanisms. Among such genes, we focused on clusterin because a link between clusterin overexpression and resistance to androgenic withdrawal (6), radiotherapy and chemotherapy has already been reported in prostate cancer (11), but also in breast cancer cells treated with trastuzumab or paclitaxel $(7,12)$. Interestingly, in different tumour types, clusterin overexpression has also been associated with disease extension and progression or with hallmarks of aggressiveness like negative estrogen and progesterone receptor status in breast cancer (13).

The cytoplasmic form of clusterin, when secreted, acts like a cytoprotective protein but its specific function remains unclear. It has been supposed that clusterin could have a chaperone-like activity because it binds to toxic molecules in the extracellular environment protecting cellular membrane integrity. In support of this hypothesis, there is the evidence that the clusterin promoter contains a sequence of $14 \mathrm{bp}$ recognised by the transcription factor heat shock factor 1 (14). In contrast, nuclear clusterin, an alternative splicing product 
lacking exon 2, functions as a pro-apoptotic protein, although its specific role and expression in cancer cells remains poorly defined.

In the present study, we investigated whether knockdown of clusterin attained with two siRNAs specifically targeted at the nuclear and cytoplasmic form (CLU-III) or exclusively at the anti-apoptotic cytoplasmic secreted form (CLU-V) has an effect on basal cell growth of three $\mathrm{ER}^{+}$breast cancer cell lines with a different sensitivity profile to antiestrogens. After having demonstrated the feasibility of effectively downregulating clusterin, we asked whether such a modification of cells could, in fact, alter their response pattern to treatment with antiestrogens.

Effectiveness of the silencing procedure was checked only on the cytoplasmic form, as this is the antiapoptotic one likely to interfere with treatment to antiestrogens, after separating nuclei from cytoplasm to avoid any contamination by the nuclear pro-apoptotic form. We observed that resistant breast cancer cells were characterised by higher protein levels of secreted clusterin than the sensitive cell line. This observation suggests that constitutive expression of clusterin could be indicative of sensitivity to antiestrogen treatment. Furthermore, we observed that clusterin levels increased after treatment with antiestrogens, a finding already reported in the literature in the case of other types of treatment (15), paclitaxel or simply estrogen withdrawal. Such treatmentinduced up-regulation of clusterin was independent of the constitutive sensitivity profile of the cell line as it occurred consistently on all three cell lines, although to a lesser extent in T47D cells. However, both siRNAs were effective in almost completely down-regulating the clusterin protein levels.

In the fairly sensitive cell line $\mathrm{MCF}-7$, up-regulation of CLU could be interpreted as an adaptive survival mechanism adopted by the cell to counteract growth inhibition effects. In fact, although MCF-7 cells were sensitive to toremifene and tamoxifen (at different degrees depending upon the length of the treatment), we were unable to observe any apoptosis (data not shown). Such an up-regulation has already been observed in other studies $(16,17)$ and might represent a general mechanism of defence of cancer cells towards cytostatic drugs. This hypothesis is supported by other studies demonstrating that clusterin is up-regulated during conditions of cell stress, similarly to that observed in breast cancer cells following trastuzumab treatment (7) and in prostate cancer cells after androgen ablation (18). Moreover, it has been demonstrated that overexpression of clusterin by stable transfection protects human cancer cells from apoptosis induced by different triggers, like radiotherapy (19) and tumour necrosis factor $\alpha$ (15). In vivo, this adaptive overexpression could increase cell survival, thereby promoting progression and acquisition of genomic instability.

We then investigated whether silencing of clusterin could influence cell proliferation and could increase the responsivity of breast cancer cell lines to tamoxifen and toremifene. We first observed that silencing of clusterin was sufficient to cause a statistically significant inhibition of cell growth, but only in the resistant cell line T47D expressing a high level of the protein. Comparable effects were obtained using both siRNAs, although the inhibition was stronger when only the cytoplasmic and not the pro-apoptotic form was downregulated, as happens with the treatment with CLU-V. Moreover, down-regulation of clusterin was able to restore sensitivity to toremifene in the resistant cell line, and once again the effects were more evident with siRNA CLU-V. Unfortunately, we did not obtain the same increase in sensitivity when T47D cells were treated with tamoxifen, probably because of their higher resistance and stronger up-regulation of clusterin by tamoxifen than toremifene.

In cells sensitive or only partially sensitive to antiestrogens, which are characterised by lower levels of clusterin than resistant cells, targeting of clusterin did not significantly modify cell growth under basal conditions or increase cell sensitivity to the treatment. We consistently observed a modest, not statistically significant drop in cell growth when the siRNA treatment was added to the antiestrogen. However, in resistant cells, which exhibited high clusterin levels, downregulation of the latter was effective in modulating response to treatment. Such results are in agreement with previous studies showing that clusterin silencing enhanced sensitivity to different therapies in distinct tumour types. After CLU silencing through siRNA and antisense strategies, cervical cancer cells expressing high levels of clusterin were reported to become more sensitive to paclitaxel (20), whereas breast cancer cells increased their response to the HER2-targeted monoclonal antibody trastuzumab (7) and chemotherapy (12).

In the present study, we demonstrated the feasibility of directly identifying in clinical studies, by gene profiling, genes which might represent good candidates for the improvement of treatment efficacy. However, the biological validation of one of these genes, clusterin, gave only partially successful results, since in sensitive cell lines down-regulation of clusterin did not improve treatment as expected. Nonetheless, the observation that by down-regulating clusterin an $\mathrm{ER}^{+}$cell line resistant to selective estrogen receptor modulators could be made sensitive to toremifene represents an interesting contribution that should be prospectively confirmed by determining clusterin expression in addition to ER before starting treatment with antiestrogens. Such results, if confirmed, could provide a rationale for modulating sensitivity to antiestrogens by combining traditional therapy with strategies targeting clusterin in vivo.

\section{Acknowledgements}

This study was supported by the Italian Association for Cancer Research (AIRC) and Special Grant 'Ricerca Finalizzata' from the Italian Health Ministry.

\section{References}

1. Gee JM, Robertson JF, Gutteridge E, Ellis IO, Pinder SE, Rubini M and Nicholson RI: Epidermal growth factor receptor/ HER2/insulin-like growth factor receptor signalling and oestrogen receptor activity in clinical breast cancer. Endocr Relat Cancer 12: S99-S111, 2005.

2. Cappelletti V, Gariboldi M, De Cecco L, Toffanin S, Reid JF, Lusa L, Bajetta E, Celio L, Greco M, Fabbri A, Pierotti MA and Daidone MG: Patterns and changes in gene expression following neo-adjuvant anti-estrogen treatment in ER-positive breast cancer. Endocr Relat Cancer 15: 439-449, 2008. 
3. Cappelletti V, Celio L, Bajetta E, Allevi A, Longarini R, Miodini P, Villa R, Fabbri A Mariani L, Giovanazzi R, Galante E, Greco $\mathrm{M}$ and Daidone MG: Prospective evaluation of estrogen receptor-beta in predicting response to neoadjuvant antiestrogen therapy in elderly breast cancer patients. Endocr Relat Cancer 11: 761-770, 2004.

4. Shannon B, Seifert M, Leskov K, Willis J, Boothman D, Tilgen W and Reichrath J: Challenge and promise: roles for CLU in pathogenesis, progression and therapy of cancer. Cell Death Differ 13: 12-19, 2006.

5. Pajak B and Orzechowski A: Overview how adenocarcinoma cancer cells avoid immune- and chemotherapy-induced apoptosis. Adv Med Sci 51: 39-45, 2006.

6. Gleave $\mathrm{M}$ and Miyake $\mathrm{H}$ : Use of antisense oligonucleotides targeting the cytoprotective gene, clusterin, to enhance androgenand chemo-sensitivity in prostate cancer. World J Urol 23: 38-46, 2005.

7. Biroccio A, D'Angel C, Jansen B, Gleave ME and Zupi G: Antisense CLU oligonucleotides increase the response of HER-2 gene amplified breast cancer cells to trastuzumab. J Cell Physiol 204: 463-469, 2005.

8. July LV, Beraldi E, So A, Fazil L, English JC and Gleave ME: Nucleotide-based therapies targeting CLU chemosensitized human lung adenocarcinoma cells both in vitro and in vivo. Mol Cancer Ther 3: 223-232, 2004.

9. Miyake H, Hara I, Kamidono S and Gleave ME: Synergistic chemosensitization and inhibition of tumor growth and metastasis by the antisense oligodeoxynucleotide targeting CLU gene in a human bladder cancer model. Clin Cancer Res 7: 4245-4252, 2001.

10. Trougakos IP, So A, Jansen B, Gleave ME and Gonos ES: Silencing expression of the clusterin/apolipoprotein $\mathrm{J}$ gene in human cancer cells using small interfering RNA induces spontaneous apoptosis, reduced growth ability, and cell sensitization to genotoxic and oxidative stress. Cancer Res 64: 1834-1842, 2004.

11. Zellweger T, Kiyama S, Chi K, Miyake H, Adomat H, Skov S and Gleave M: Overexpression of the cytoprotective protein CLU decreases radiosensitivity in the human $\mathrm{LNCaP}$ prostate tumor model. BJU Int 92: 463-469, 2003.
12. So A, Sinnemann S, Huntsman D, Fazli L and Gleave M: Knockdown of the cytoprotective chaperone, clusterin, chemosensitizies human breast cancer cells both in vitro and in vivo. Mol Cancer Ther 4: 1837-1849, 2005.

13. Redondo M, Villar E, Torrez-Munoz J, Tellez T, Morell M and Petito C: Overexpression of CLU in human breast carcinoma. Am J Pathol 157: 393-399, 2000.

14. Humphreys DT, Carver JA, Easterbrook-Smith SB and Wilson MR: CLU has chaperone-like activity similar to that of small heat shock proteins. J Biol Chem 274: 6875-6881, 1999.

15. Sensibar JA, Sutkowski DM, Raffo A, Buttyan R, Griswold MD, Sylvester SR, Kozlowski JM and Lee C: Prevention of cell death induced by tumor necrosis factor alpha in LNCaP cells by overexpression of sulfated glycoprotein-2 (clusterin). Cancer Res 55: 2431-2437, 1995

16. Warri AM, Huovinen RL, Laine AM, Martikainen PM and Harkonen PL: Apoptosis in toremifene-induced growth inhibition of human breast cancer cells in vivo and in vitro. J Natl Cancer Inst 85: 1412-1418, 1993 .

17. Chen H, Tritton TR, Kenny N, Absher M and Chiu JF: Tamoxifen induces TGF-beta 1 activity a.nd apoptosis of human MCF-7 breast cancer cells in vitro. J Cell Biochem 61: 9-17, 1996.

18. July LV, Akbari M, Zellweger T, Jones EC, Goldenberg SL and Gleave ME: CLU expression is significantly enhanced in prostate cancer cells following androgen withdrawal therapy. Prostate 50: 179-188, 2002.

19. Zellweger T, Chi K, Miyake H, Adomat H, Kiyama S, Skov K and Gleave ME: Enhanced radiation sensitivity in prostate cancer by inhibition of the cell survival protein clusterin. Clin Cancer Res 8: 3276-3284, 2002.

20. Park DC, Yeo SG, Shin EY, Mok SC and Kim DH: CLU confers paclitaxel resistance in cervical cancer. Gynecol Oncol 103: 996-1000, 2006 